\title{
Pandemic created clinical eclipse: hemosiderosis versus COVID-19 pneumonia
}

\author{
Vijayanand Palanisamy ${ }^{1}$ (1) $\cdot$ Pradeep Ramkoomar $^{1} \cdot$ Shilpa Shree $^{1} \cdot$ Mithun Sundararaaja Ravikumar $^{1}$
}

Received: 12 August 2021 / Revised: 24 September 2021 / Accepted: 27 September 2021 / Published online: 4 November 2021

(C) Indian Association of Cardiovascular-Thoracic Surgeons 2021

\begin{abstract}
In the severe acute respiratory syndrome coronavirus-2 (SARS-CoV-2) pandemic, the physician finds difficulty in differentiating the symptoms due to cardiac disease from that of SARS-CoV-2. We would like to present one such mystified situation (hemosiderosis versus SARS-CoV-2 pneumonia) we encountered.
\end{abstract}

Keywords Hemosiderosis · SARS-CoV-2 pneumonia

\section{Case report}

A 37-year-old female, with a known history of rheumatic mitral stenosis for the past 10 years, presented with complaints of progressive breathlessness, chest pain, and palpitation on minimal exertion.

While evaluating for surgical fitness, on computed tomography, the coronavirus disease 2019 Reporting and Data System (CO-RADS) score of 5 (Fig. 1) and chest roentgenogram (Fig. 2A) findings were suggestive of severe acute respiratory syndrome coronavirus-2 (SARS-CoV-2), while reverse transcriptase-polymerase chain reaction (RT-PCR) was negative (Fig. 1). Surgery was deferred to a later date. While evaluating 15 days later, RT-PCR was still negative and both the immunoglobulin $\mathrm{G}(\mathrm{IgG})$ and immunoglobulin M (IgM) SARS-CoV-2 antibodies were also negative.

She underwent mitral valve replacement successfully. A postoperative chest roentgenogram was evident for the presence of hemosiderosis (Fig. 2B). Dissecting retrograde, we concluded that hemosiderosis with failure features was mocked with SARS-CoV-2 features.

Patchy areas of ground-glass opacity or consolidation in selected lung zones may be earlier radiological features of secondary pulmonary hemosiderosis, which may be reversible, once pulmonary venous hypertension is relieved. A

Vijayanand Palanisamy

vetri86@gmail.com

1 Department of Cardiac Surgery, Institute of Cardiovascular Diseases, The Madras Medical Mission, 4A, Dr. J.J. Nagar, Mogappair, Chennai 600037, Tamil Nadu, India study conducted in nine centers of the UK [1] revealed a higher mortality rate $(24.5 \%$ vs $3.5 \%, p<0.0001)$ and longer postoperative stay ( 11 days vs 6 days, $p=0.001$ ). In this pandemic, weighing benefit versus risk, it is better to wait, when there is no concrete evidence to rule out SARS-CoV-2.

The two pulmonary conditions which have nearly a similar pattern to hemosiderosis are nonspecific interstitial pneumonitis and cryptogenic organizing pneumonia [2]. In nonspecific interstitial pneumonitis, there will be a predominant lower zone involvement and a hallmark subpleural sparing in the computed tomography (CT). In cryptogenic organizing pneumonia, the characteristic feature will be the reverse halo sign but it will be seen only in $20 \%$ of the patients. Perilobular thickening and migratory opacities are helpful differentiating features.

Author contribution Vijayanand Palanisamy-concept/drafting the article. Pradeep Ramkoomar-revision. Shilpa Shree-drafting. Mithun Sundararaaja Ravikumar-revision. All authors listed meet the authorship criteria according to the latest guidelines of the International Committee of Medical Journal Editors and that all authors are in agreement with the manuscript. All authors of this research paper have directly participated in the planning, execution, or analysis of this study.

Funding None. 
Fig. 1 A, B Chest computed tomography scan showing bilateral diffuse ground-glass opacity with patchy lung changes in mid and lower lung zones
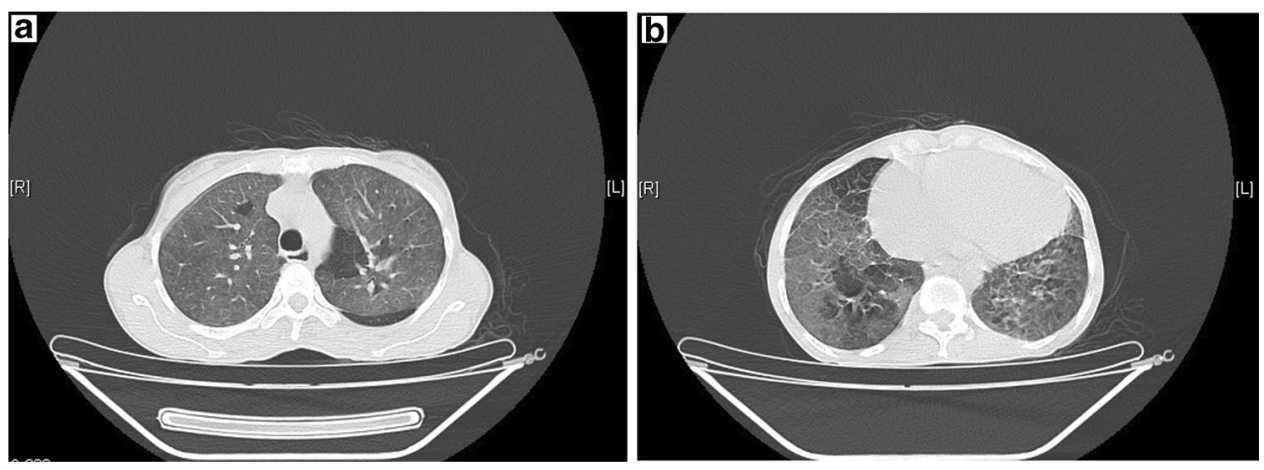

Fig. 2 Chest roentgenogram. (A) Preoperative images showing bilateral reticulonodular opacities with patchy lung changes involving mid and lower zones. (B) Postoperative image showing reversal of patchy lung changes and revealing features of hemosiderosis (bilateral reticulonodular opacity)
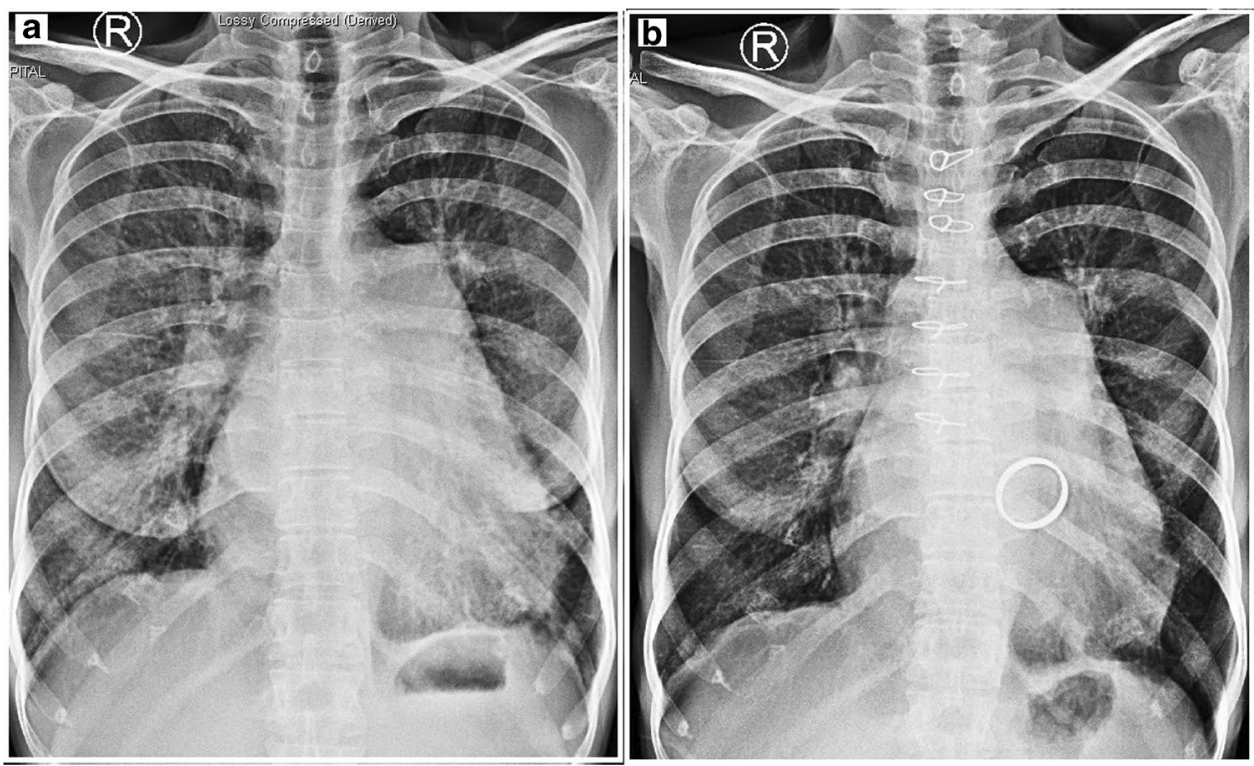

\section{Declarations}

Institutional review board approval Institutional review board approval has been obtained for the publishing of patient profile and this article.

Informed consent Obtained to publish this report.

Statement of human and animal rights Not Applicable.

Conflict of interest The authors declare no competing interests.

\section{References}

1. Sanders J, Akowuah E, Cooper J, et al. Cardiac surgery outcome during the COVID-19 pandemic: a retrospective review of the early experience in nine UK centres. J Cardiothorac Surg. 2021;16:43. https://doi.org/10.1186/s13019-021-01424-y.

2. Parekh M, Donuru A, Balasubramanya R, Kapur S. Review of the chest CT differential diagnosis of ground-glass opacities in the COVID era. Radiology. 2020;297:E289-302. https://doi.org/ 10.1148/radiol.2020202504.

Publisher's note Springer Nature remains neutral with regard to jurisdictional claims in published maps and institutional affiliations. 